\title{
TOPOLOGICAL TYPE OF LIMIT LAMINATIONS OF EMBEDDED MINIMAL DISKS
}

\author{
JACOB BERNSTEIN AND GIUSEPPE TINAGLIA
}

\begin{abstract}
We consider two natural classes of minimal laminations in threemanifolds. Both classes may be thought of as limits - in different senses - of embedded minimal disks. In both cases, we prove that, under a natural geometric assumption on the three-manifold, the leaves of these laminations are topologically either disks, annuli or Möbius bands. This answers a question posed by Hoffman and White.
\end{abstract}

\section{INTRODUCTION}

Let $\Omega$ be a a fixed Riemannian three-manifold. Suppose that $\Omega_{i} \subset \Omega$ is an increasing sequence of open sets with $\Omega=\bigcup \Omega_{i}$ and that $\Sigma_{i}$ are minimal surfaces properly embedded in $\Omega_{i}$. We say that the curvatures of the $\Sigma_{i}$ blow up at a point $p \in \Omega$ if there exists a sequence of points $p_{i} \in \Sigma_{i}$ converging to $p$ such that $\left|A_{\Sigma_{i}}\right|\left(p_{i}\right)$ becomes arbitrarily large; where $\left|A_{\Sigma}\right|$ denotes the norm of the second fundamental form of $\Sigma$. We call such $p$ a blow-up point and observe that the set, $K$, of blow-up points is closed in $\Omega$. The points of $K$ are precisely the obstruction to the sequence smoothly subconverging. Indeed, up to passing to a subsequence, the $\Sigma_{i} \backslash K$ converge on compact subsets of $\Omega \backslash K$ to a smooth proper minimal lamination $\mathcal{L}$ in $\Omega \backslash K$ - see [7, Appendix B]. Recall, a lamination is a foliation that need not fill space. We call the quadruple $(\Omega, K, \mathcal{L}, \mathcal{S})$ a minimal surface sequence. A natural question is:

In a minimal surface sequence, what singular sets, $K$, and limit laminations, $\mathcal{L}$, can arise?

Work of Anderson [1] and White [23], answers this question when the total extrinsic curvatures of the surfaces $\Sigma_{i}$ - i.e., $\int_{\Sigma_{i}}\left|A_{\Sigma_{i}}\right|^{2}-$ are uniformly bounded. In this case, $K$ is finite and $\mathcal{L}$ extends smoothly across $K$. In general, without such a strong assumption on the geometry of the surfaces in the sequence one does not expect such a complete answer.

Remarkably, when the $\Sigma_{i}$ are assumed only to be disks, an elegant story also emerges - we call such minimal surface sequences, minimal disk sequences. In a series of papers [5]-8], Colding and Minicozzi extensively studied these sequences and proved deep structural results about both their singular sets, $K$, and limit laminations, $\mathcal{L}$. Specifically, they showed that $K$ must be contained in a Lipschitz

The first author was partially supported by the EPSRC Programme Grant entitled "Singularities of Geometric Partial Differential Equations" grant number EP/K00865X/1. The second author was partially supported by EPSRC grant no. EP/L003163/1. 
curve and that for any point $p \in K$ there exists a leaf of $\mathcal{L}$ that extends smoothly across $p$. When $\Omega=\mathbb{R}^{3}$, Colding and Minicozzi further showed that either $K=\emptyset$ or $\mathcal{L}$ is a foliation of $\mathbb{R}^{3} \backslash K$ by parallel planes and that $K$ consists of a connected Lipschitz curve which meets the leaves of $\mathcal{L}$ transversely. Using this result, Meeks and Rosenberg showed in [16] that the helicoid is the unique non-flat properly embedded minimal disk in $\mathbb{R}^{3}$ - see also [2]. This uniqueness was then used by Meeks [15] to prove that if $\Omega=\mathbb{R}^{3}$ and $K \neq \emptyset$, then $K$ is a line orthogonal to the leaves of $\mathcal{L}$. This is precisely the limit of a sequence of rescalings of a helicoid.

An example constructed by Colding and Minicozzi in [4], illustrates how for general regions such a simple and complete description of the limit lamination does not hold. Specifically, they constructed a sequence of properly embedded minimal disks in the unit ball $B_{1}$ of $\mathbb{R}^{3}$ which has $K=\{0\}$ and whose limit lamination consists of three leaves - two are non-proper and spiralling into the third which is the punctured unit disk in the $x_{3}$-plane. Inspired by this, a plethora of examples have now been constructed which show that the singular set $K$ can consist of any closed subset of a line, see [9, 11-13]. Likewise, Meeks and Weber [17] have given examples where $K$ is curved. Strikingly, Hoffman and White [10] have also constructed minimal disk sequences in which $K=\emptyset$ and the limit lamination $\mathcal{L}$ has a leaf which is a proper annulus in $\Omega$. In all examples the leaves are either topologically disks or annuli. This motivated Hoffman and White to ask in [10]:

Can a surface of positive genus occur as a leaf of the lamination $\mathcal{L}$ of a minimal disk sequence? A planar domain with more than two holes?

In this paper we show that, under natural geometric assumptions on $\Omega$, the answer to both questions is no. That is, the leaves of $\mathcal{L}$ must be either disks or annuli.

Theorem 1.1. Let $\Omega$ be the interior of a compact oriented three-manifold $N=$ $\bar{\Omega}$ with mean-convex boundary. If $\Omega$ contains no closed minimal surfaces and $(\Omega, K, \mathcal{L}, \mathcal{S})$ is a minimal disk sequence, then the leaves of $\mathcal{L}$ are either disks or annuli. Furthermore, if $L$ is a leaf of $\mathcal{L}$ with the property that $\bar{L}$ - the closure in $\Omega$ of $L-i$ a properly embedded minimal surface, then $\bar{L}$ is either a disk or it is an annulus which is disjoint from $K$.

The theorem is proved by realizing the disks in the sequence $\mathcal{S}$ as effective universal covers of the leaves of $\mathcal{L}$. Geometric considerations - specifically the fact that the disks are embedded - strongly restrict these covers and this restricts the topology of the leaves as claimed. Our proof uses relatively elementary topological and geometric properties of embedded minimal disks - in particular, we do not directly use the deep results of $[5-\sqrt{8}]$.

Simple examples show that it is possible for the leaves of $\mathcal{L}$ to be planar domains with more than two holes when the boundary is not assumed to be mean-convex. Nevertheless, the methods of the present paper continue to show that the leaves are genus zero. However, as treating this more general case introduces several technical points, we do not persue it.

We further remark that it is unclear whether the condition that $\Omega$ admits no closed minimal surface is necessary. To better understand this question we consider 
also the topological type of leaves of a different - though related - class of minimal laminations. Specifically, we say that a quadruple $\left(\Omega, K, \mathcal{L}, \mathcal{L}_{0}\right)$ is a minimal disk closure if $\mathcal{L}_{0}$ is a minimal lamination in $\Omega$ all of whose leaves are disks, $K$ is a relatively closed subset of $\Omega$ and $\mathcal{L}=\overline{\mathcal{L}}_{0}$, the closure of $\mathcal{L}_{0}$ in $\Omega \backslash K$, is a proper minimal lamination in $\Omega \backslash K$.

Theorem 1.2. Let $\Omega$ be the interior of a compact oriented three-manifold with boundary $N=\bar{\Omega}$ which has mean-convex boundary. If $\Omega$ contains no closed minimal surfaces and $\left(\Omega, K, \mathcal{L}, \mathcal{L}_{0}\right)$ is a minimal disk closure, then the leaves of $\mathcal{L}$ are either topologically open subsets $\mathbb{S}^{2}$ or open subsets $\mathbb{R P}^{2}$.

Notice that the conclusions of Theorem 1.2 are weaker than those of Theorem 1.1 in two ways. First, we cannot rule out that the presence of many punctures. Secondly, we can no longer rule out the existence of one-sided leaves of $\mathcal{L}$. In Appendix A we give an example to show this weakening is unavoidable - that is, we construct a minimal disk closures whose leaves have multiple punctures and a minimal disk closure $\left(\Omega, \emptyset, \mathcal{L}, \mathcal{L}_{0}\right)$ where $\Omega$ is a solid torus and $\mathcal{L}$ contains a Möbius band as a leaf. We also give an example in Appendix $\mathrm{A}$ that shows that Theorem 1.2 is sharp - i.e., we construct a minimal disk closure $\left(\Omega, \emptyset, \mathcal{L}, \mathcal{L}_{0}\right)$ for which one leaf of $\mathcal{L}$ is a torus.

\section{NOTATION}

Fix a smooth oriented Riemannian three-manifold $(\Omega, g)$. We denote by dist ${ }^{\Omega}$ the distance function on $\Omega$ and by $\exp ^{\Omega}$ the exponential map. Hence,

$$
\exp _{p}^{\Omega}: B_{r} \rightarrow \mathcal{B}_{r}(p)
$$

where $B_{r}$ is the usual euclidean ball in $\mathbb{R}^{3}$ centered at the origin and $\mathcal{B}_{r}(p)$ is the geodesic ball in $\Omega$. A subset $\Sigma \subset \Omega$ is an embedded smooth surface if for each point $p \in \Sigma$ there is a radius $r_{p}>0$ and diffeomorphism $\phi_{p}: \mathcal{B}_{r_{p}}(p) \rightarrow B_{1}$ so that $\mathbb{D}_{1}=\phi_{p}\left(\Sigma \cap \mathcal{B}_{r_{p}}(p)\right)$. Here, $\mathbb{D}_{1}=B_{1} \cap\left\{x_{3}=0\right\} \subset B_{1}$ is the unit disk. Such $\Sigma$ is said to be proper in $\Omega$ if it is a closed subset of $\Omega$, that is, $\bar{\Sigma}=\Sigma$.

For an embedded smooth surface, $\Sigma$, we write

$$
\exp ^{\perp}: N \Sigma \rightarrow \Omega
$$

for the normal exponential map where here $N \Sigma$ is the normal bundle. If $N \Sigma$ is trivial then we say that $\Sigma$ is two-sided, otherwise we say that $\Sigma$ is one-sided. As $\Omega$ is oriented, $\Sigma$ is two-sided if and only if $\Sigma$ is orientable. For a subset $U \subset N \Sigma$ set

$$
\mathcal{N}_{U}(\Sigma):=\exp ^{\perp}(U)
$$

The set $\mathcal{N}_{U}(\Sigma)$ is regular if there is an open set $V$ with $U \subset V$ so that $\exp ^{\perp}: V \rightarrow$ $\mathcal{N}_{V}(\Sigma)$ is a diffeomorphism. If $\mathcal{N}_{U}(\Sigma)$ is regular, then the map $\Pi_{\Sigma}: \mathcal{N}_{U}(\Sigma) \rightarrow \Sigma$ given by nearest point projection is smooth and for any $(q, \mathbf{v}) \in T \mathcal{N}_{U}(\Sigma)$, there is a natural splitting $\mathbf{v}=\mathbf{v}^{\perp}+\mathbf{v}^{\top}$, where $\mathbf{v}^{\perp}$ is orthogonal to $\mathbf{v}^{\top}$ and $\mathbf{v}^{\perp}$ is tangent to the fibers of $\Pi_{\Sigma}$. We say such $\mathbf{v}$ is $\delta$-parallel to $\Sigma$ if

$$
\left|\mathbf{v}^{\perp}\right| \leq \delta|\mathbf{v}| \text { and } \frac{1}{1+\delta}\left|\mathbf{v}^{\top}\right| \leq\left|d\left(\Pi_{\Sigma}\right)_{q}(\mathbf{v})\right| \leq(1+\delta)\left|\mathbf{v}^{\top}\right| .
$$


Given $\epsilon>0$ we set $U_{\epsilon}=\{(p, \mathbf{v}) \in N \Sigma:|\mathbf{v}|<\epsilon\}$ and define $\mathcal{N}_{\epsilon}(\Sigma)$, the $\epsilon$ neighborhood of $\Sigma$, to be $\mathcal{N}_{U_{\epsilon}}(\Sigma)$. If $\Sigma$ is an embedded smooth surface and $\Sigma_{0}$ is a pre-compact subset, then there is an $\epsilon>0$ so that $\mathcal{N}_{\epsilon}\left(\Sigma_{0}\right)$ is regular.

Given a fixed embedded surface $\Sigma$ and $\delta \geq 0$ we say that another embedded smooth surface $\Gamma$ is a smooth $\delta$-graph over $\Sigma$ if there is an $\epsilon>0$ so that the following holds:

(1) $\mathcal{N}_{\epsilon}(\Sigma)$ is a regular $\epsilon$-neighborhood of $\Sigma$;

(2) Either $\Gamma$ is a proper subset of $\mathcal{N}_{\epsilon}(\Sigma)$ or $\Gamma$ is a proper subset of $\mathcal{N}_{\epsilon}(\Sigma) \backslash \Sigma$;

(3) $\operatorname{Each}(q, \mathbf{v}) \in T \Gamma$ is $\delta$-parallel to $\Sigma$.

We say that a smooth $\delta$-graph over $\Sigma, \Gamma$, is a smooth $\delta$-cover of $\Sigma$ if it is connected and

$$
\Pi_{\Sigma}(\Gamma)=\Sigma \text {. }
$$

Let $\gamma:[0,1] \rightarrow \Sigma$ be a $C^{1}$ curve in $\Sigma$. We will also denote the image of such $\gamma$ by $\gamma$. We say that a curve $\widetilde{\gamma}:[0,1] \rightarrow \mathcal{N}_{\delta}(\gamma)$ is a $\delta$-lift of $\gamma$ if $\mathcal{N}_{\delta}(\gamma)$ is regular, $\Pi_{\Sigma} \circ \widetilde{\gamma}=\gamma$ and for each $t \in[0,1],\left(\widetilde{\gamma}(t), \widetilde{\gamma}^{\prime}(t)\right)$ is $\delta$-parallel to $\Sigma$. This definition extends to piece-wise $C^{1}$ curves in an obvious manner.

\section{Minimal Laminations}

We recall some facts about laminations.

Definition 3.1. A subset $\mathcal{L} \subset \Omega$ is a smooth lamination if for each $p \in \mathcal{L}$, there is a radius $r_{p}>0$, maps $\phi_{p}, \psi_{p}: \mathcal{B}_{r_{p}}(p) \rightarrow B_{1} \subset \mathbb{R}^{3}$ and a closed set $0 \in T_{p} \subset$ $(-1,1)$ so that:

(1) $\phi_{p}(p)=\psi_{p}(p)=0$;

(2) $\phi_{p}$ is a smooth diffeomorphism and $\mathbb{D}_{1} \subset \phi_{p}\left(\mathcal{L} \cap \mathcal{B}_{r_{p}}(p)\right)$;

(3) $\psi_{p}$ is a Lipschitz diffeomorphism and $B_{1} \cap\left\{x_{3}=t\right\}_{t \in T_{p}}=\psi_{p}\left(\mathcal{L} \cap \mathcal{B}_{r_{p}}(p)\right)$;

(4) $\psi_{p}^{-1}\left(\mathbb{D}_{1}\right)=\phi_{p}^{-1}\left(\mathbb{D}_{1}\right)$.

We refer to maps $\phi_{p}$ satisfying (11) and (2) as smoothing maps of $\mathcal{L}$ and to maps $\psi_{p}$ satisfying (1) and (3) as straightening maps of $\mathcal{L}$.

A smooth lamination $\mathcal{L} \subset \Omega$ is proper in $\Omega$ if it is closed - i.e. $\overline{\mathcal{L}}=\mathcal{L}$. Any embedded smooth surface is a smooth lamination, which is proper if and only if the surface is.

Definition 3.2. Let $\mathcal{L} \subset \Omega$ be a non-empty smooth lamination. A subset $L \subset \mathcal{L}$ is a leaf of $\mathcal{L}$ if it is a connected, embedded surface and for any $p \in L$, there is an $r_{p}>0$ and a smoothing map $\phi_{p}$ so that $\mathbb{D}_{1}=\phi_{p}\left(L \cap \mathcal{B}_{r_{p}}(p)\right)$. For each $p \in \mathcal{L}$, let $L_{p}$ be the unique leaf of $\mathcal{L}$ containing $p$.

Definition 3.3. A smooth lamination $\mathcal{L}$ is a minimal lamination if each leaf is minimal.

A sequence $\left\{\Omega_{i}\right\}$ of open subsets of $\Omega$ exhausts $\Omega$ if $\Omega_{i} \subset \Omega_{i+1}$ and $\Omega=$ $\bigcup_{i=1}^{\infty} \Omega_{i}$. 
Definition 3.4. Suppose the sequence $\left\{\Omega_{i}\right\}$ exhausts $\Omega$ and that $\mathcal{L}_{i}$ are smooth proper laminations in $\Omega_{i}$. For any $0<\alpha<1$, the $\mathcal{L}_{i}$ converge in $C_{l o c}^{\infty, \alpha}(\Omega)$ to $\mathcal{L}$, a proper smooth lamination in $\Omega$, provided:

(1) The sets $\mathcal{L}_{i}$ converge to $\mathcal{L}$ in pointed Gromov-Hausdorff distance;

(2) Smoothing maps of the $\mathcal{L}_{i}$ converge in $C^{\infty}$ to smoothing maps of $\mathcal{L}$. That is, for each $p \in \mathcal{L}$ there is an $r_{p}>0$ and an $i_{p}>0$ so that: for $i>i_{p}$, $\mathcal{B}_{r_{p}}(p) \subset \Omega_{i}$ and for all $p_{i} \in \mathcal{B}_{\frac{1}{4} r_{p}}(p) \cap \mathcal{L}_{i}$ converging to $p$, there are $r_{p_{i}} \geq r_{p}$ and smoothing maps $\phi_{i}: \mathcal{B}_{\frac{1}{2} r_{p}}\left(p_{i}\right) \rightarrow B_{1}$ of the $\mathcal{L}_{i}$ converging in $C_{\text {loc }}^{\infty}\left(\mathcal{B}_{\frac{1}{4} r_{p}}(p)\right)$ to a smoothing map $\phi_{p}: \mathcal{B}_{\frac{1}{4} r_{p}}(p) \rightarrow B_{1}$ of $\mathcal{L}$.

(3) Straightening maps of the $\mathcal{L}_{i}$ converge in $C^{\alpha}$ to straightening maps of $\mathcal{L}$. That is, for each $p \in \mathcal{L}$ there is an $r_{p}>0$ and an $i_{p}>0$ so that: for $i>i_{p}$, $\mathcal{B}_{r_{p}}(p) \subset \Omega_{i}$ and for all $p_{i} \in \mathcal{B}_{\frac{1}{4} r_{p}}(p) \cap \mathcal{L}_{i}$ converging to $p$, there are $r_{p_{i}} \geq r_{p}$ and straightening maps $\psi_{i}: \mathcal{B}_{\frac{1}{2} r_{p}}\left(p_{i}\right) \rightarrow B_{1}$ of the $\mathcal{L}_{i}$ converging in $C_{\text {loc }}^{\alpha}\left(\mathcal{B}_{\frac{1}{4} r_{p}}(p)\right)$ to a straightening map $\psi_{p}: \mathcal{B}_{\frac{1}{4} r_{p}}(p) \rightarrow B_{1}$ of $\mathcal{L}$.

The following is the natural compactness result for sequences of properly embedded minimal surfaces with uniformly bounded second fundamental form see [7, Appendix B] for a proof.

Theorem 3.5. Suppose that $\left\{\Omega_{i}\right\}$ exhausts $\Omega$ and that $\Sigma_{i}$ are properly embedded smooth minimal surfaces in $\Omega_{i}$. If for each compact subset $U$ of $\Omega$ there is a constant $C(U)<\infty$ so that when $U \subset \Omega_{i}$

$$
\sup _{U \cap \Sigma_{i}}\left|A_{\Sigma_{i}}\right| \leq C(U)
$$

then, for any $0<\alpha<1$, up to passing to a subsequence, the $\Sigma_{i}$ converge in $C_{\text {loc }}^{\infty, \alpha}(\Omega)$ to a smooth proper minimal lamination $\mathcal{L}$ in $\Omega$.

Remark 3.6. While the straightening maps converge in $C^{\alpha}$, their Lipschitz norms are uniformly bounded on compact subsets of $\Omega$. This follows from the Harnack inequality and is used in the proof of Theorem 3.5]- see (B.3) and (B.5) of [7] and also [21, Theorem 1.1].

Suppose that $\left\{\Omega_{i}\right\}$ exhausts $\Omega$ and that $\Sigma_{i}$ are properly embedded smooth minimal surfaces in $\Omega_{i}$. In light of Theorem 3.5, we define the regular points of the sequence $\mathcal{S}=\left\{\Sigma_{i}\right\}$ to be the set of points

$$
\operatorname{reg}(\mathcal{S}):=\left\{p \in \Omega: \exists \rho>0 \text { s.t. } \limsup _{i \rightarrow \infty} \sup _{B_{\rho}(p) \cap \Sigma_{i}}\left|A_{\Sigma_{i}}\right|<\infty\right\}
$$

and the singular points of $\mathcal{S}$ to be the set

$$
\operatorname{sing}(\mathcal{S}):=\left\{p \in \Omega: \forall \rho>0, \liminf _{i \rightarrow \infty} \sup _{B_{\rho}(p) \cap \Sigma_{i}}\left|A_{\Sigma_{i}}\right|=\infty\right\} .
$$

Clearly, $\operatorname{reg}(\mathcal{S})$ is an open subset of $\Omega$ while $\operatorname{sing}(\mathcal{S})$ is closed in $\Omega$. In general, $\operatorname{sing}(\mathcal{S})$ is a strict subset of $\Omega \backslash \operatorname{reg}(\mathcal{S})$. However, an elementary argument - see [7, Lemma I.1.4] - implies that there is a subsequence $\mathcal{S}^{\prime}$ of $\mathcal{S}$ so that $\Omega=\operatorname{reg}\left(\mathcal{S}^{\prime}\right) \cup$ 
$\operatorname{sing}\left(\mathcal{S}^{\prime}\right)$. From now on we consider only sequences for which this decomposition holds.

We say that $\mathcal{L}$ is the limit lamination of $\mathcal{S}$ if for some $\alpha>0, \Sigma_{i} \rightarrow \mathcal{L}$ in $C_{l o c}^{\infty, \alpha}(\operatorname{reg}(\mathcal{S}))$. Theorem 3.5 implies that, up to passing to a subsequence, any sequence $\mathcal{S}$, possesses a limit lamination $\mathcal{L}$. Inspired by [22], we make the following definition:

Definition 3.7. We say a quadruple $(\Omega, K, \mathcal{L}, \mathcal{S})$ consisting of

(1) A Riemannian three-manifold $\Omega$ exhausted by $\left\{\Omega_{i}\right\}$;

(2) A closed set $K \subset \Omega$;

(3) A proper smooth minimal lamination $\mathcal{L}$ in $\Omega \backslash K$; and

(4) A sequence $\mathcal{S}=\left\{\Sigma_{i}\right\}$ of properly embedded minimal surfaces $\Sigma_{i}$ in $\Omega_{i}$, is a minimal surface sequence if

(1) $\operatorname{sing}(\mathcal{S})=K$; and

(2) $\Sigma_{i} \backslash K$ converge in $C_{\text {loc }}^{\infty, \alpha}(\Omega \backslash K)$ to $\mathcal{L}$ for some $0<\alpha<1$.

If all the surfaces in $\mathcal{S}$ are disks, then we say this is a minimal disk sequence.

The work of Colding and Minicozzi [5- 8 ] implies that if $(\Omega, K, \mathcal{L}, \mathcal{S})$ is a minimal disk sequence, then $K$ and $\mathcal{L}$ have a great deal of structure. We say a leaf $L$ of $\mathcal{L}$ is regular at $p \in K$ if $p \in \bar{L}$, the closure of $L$ in $\Omega$, and there is an $r>0$ so that $\mathcal{B}_{r}(p) \cap \bar{L}$ is an embedded smooth surface proper in $\mathcal{B}_{r}(p)$. Then the following holds.

Proposition 3.8. If $(\Omega, K, \mathcal{L}, \mathcal{S})$ is a minimal disk sequence, then there is an embedded one-dimensional Lipschitz curve $K^{\prime} \subset \Omega$ such that $K \subset K^{\prime}$ and

(1) If $p \in K$, then there is a leaf $L \in \mathcal{L}$ which is regular at $p$;

(2) If $L$ is regular at $p \in K$, then $\bar{L}$ meets $K$ transversely - in the strong sense that $\bar{L}$ meets $K^{\prime}$ transversely at $p$.

White [22] has shown that the regularity of $K^{\prime}$ can be taken to be $C^{1}$. Furthermore, Meeks [14] has shown that if $K=K^{\prime}$, then $K^{\prime}$ can be taken to be $C^{1,1}$.

We will consider also the following related objects.

Definition 3.9. We say a quadruple $\left(\Omega, K, \mathcal{L}, \mathcal{L}_{0}\right)$ consisting of

(1) A connected open subset $\Omega \subset N$;

(2) A closed set $K \subset \Omega$;

(3) A smooth minimal lamination $\mathcal{L}_{0}$ in $\Omega \backslash K$; and

(4) A smooth proper minimal lamination $\mathcal{L}$ in $\Omega \backslash K$,

is a minimal surface closure provided,

(1) For all $p \in K$ and $\rho>0$, $\sup _{L \in\left\{\mathcal{L}_{0}\right\}} \sup _{B_{\rho}(p) \cap L}\left|A_{L}\right|=\infty$; and

(2) $\overline{\mathcal{L}}_{0}=\mathcal{L}$, where here $\overline{\mathcal{L}}_{0}$ is the closure of $\mathcal{L}_{0}$ in $\Omega \backslash K$.

If all the leaves of $\mathcal{L}_{0}$ are disks, then we say this is a minimal disk closure.

The limit laminations $\mathcal{L}$ of minimal disk sequences and of minimal disk closures share many properties. Therefore, it is convenient to introduce the following definition. 
Definition 3.10. A smooth minimal lamination $\mathcal{L}$ in a Riemannian three-manifold $\Omega$ is a simple minimal lamination in $\Omega$ if there is a relatively closed set $K \subset \Omega$ and either

(1) $(\Omega, K, \mathcal{L}, \mathcal{S})$ is a minimal disk sequence for some $\mathcal{S}$; or

(2) $\left(\Omega, K, \mathcal{L}, \mathcal{L}_{0}\right)$ is a minimal disk closure for some $\mathcal{L}_{0}$.

\section{SiMPlE LifTS}

In order to proceed we will need a technical definition.

Definition 4.1. Let $\Sigma$ be an embedded surface in a fixed Riemannian three-manifold $\Omega$. The surface $\Sigma$ has the simple lift property if, for any $\delta>0, \gamma:[0,1] \rightarrow \Sigma$ a piece-wise $C^{1}$ curve, and open pre-compact subset $U \subset \Sigma$ with $\gamma \subset U$, there exist:

(1) A constant $\epsilon=\epsilon(U, \delta)>0$;

(2) An embedded minimal disk $\Delta$ in $\Omega$; and

(3) A $\delta$-lift of $\gamma, \widehat{\gamma}:[0,1] \rightarrow \mathcal{N}_{\delta}(U)$,

such that

(1) $\widehat{\gamma} \subset \Delta \cap \mathcal{N}_{\epsilon}(U)$;

(2) $\Delta \cap \mathcal{N}_{\epsilon}(U)$ is a $\delta$-graph over $U$;

(3) The connected component of $\Delta \cap \mathcal{N}_{\epsilon}(U)$ containing $\widehat{\gamma}$ is a $\delta$-cover of $U$.

Such $\widehat{\gamma}$ is called a simple $\delta$-lift of $\gamma$ into $\Omega$.

If $\Sigma$ has the simple lift property in $\Omega$ and $\gamma$ is a curve in $\Sigma$, then $\gamma$ has the embedded lift property if there is a a $\delta_{0}>0$ so that for all $\delta_{0}>\delta>0$, all simple $\delta$-lifts of $\gamma$ are embedded. Clearly, if $\gamma$ is an embedded curve, then it has the embedded lift property.

Throughout this paper we study the topology of minimal surfaces with the simple lift property. This is relevant to the study of the topology of a leaf of a simple minimal lamination thanks to the following proposition.

Proposition 4.2. Leaves of a simple minimal lamination in $\Omega$ have the simple lift property.

Proof. We first consider the case of a minimal disk closure. If $L$ is a leaf of $\mathcal{L}$ which is a disk, then any curve in $L$ is its own simple $\delta$-lift in any pre-compact open set of $L$ containing such curve and there is nothing to prove. If $L$ is not a disk, then $L \subset \mathcal{L} \backslash \mathcal{L}_{0}$. Hence, for any point $p \in L$ there exists a sequence of points $p_{i} \in \mathcal{L}_{0}$ so that $p_{i} \rightarrow p$.

Note first that the definition of smooth lamination - specifically the existence of Lipschitz straightening maps - implies that for each pre-compact open subset $U$ of $L$ there is a constant $C=C(U)$ so that if $1>C \lambda>0$, then, for each leaf $L^{\prime}$ of $\mathcal{L}_{0}, \mathcal{N}_{\lambda}(U) \cap L^{\prime}$ is a - possibly empty - $C \lambda$-graph over $U$. Given a curve $\gamma:[0,1] \rightarrow L$ and $U$ some pre-compact open subset of $L$ so that $\gamma \subset U$, let $l$ denote the length of $\gamma$ and let $d$ denote the diameter of $U$. For any $\delta>0$, choose $\epsilon>0$ such that $C \epsilon<\min \{1, \delta\}$. Let $\mu=\frac{3}{4} e^{-2 C(l+d)}$ and pick $L_{\mu}$ to be a leaf of $\mathcal{L}_{0}$ which satisfies $\mathcal{N}_{\mu \epsilon}(p) \cap L_{\mu} \neq \emptyset$ where here $p=\gamma(0)$. Let $\Gamma$ be a component of $L_{\mu} \cap \mathcal{N}_{\epsilon}(U)$ which contains a point $q \in \mathcal{N}_{\mu \epsilon}(p) \cap \Gamma$. 
The leaf $L_{\mu}$ is, by definition, a disk and we have chosen $\epsilon>0$ so that $L_{\mu} \cap \mathcal{N}_{\epsilon}(U)$ is a $\delta$-graph over $U$. We claim that $\Gamma$ is a $\delta$-cover of $U$ containing a $\delta$-lift of $\gamma$. This follows by showing that any curve in $U$ of length at most $2(l+d)$ starting at $p$ has a lift in $\Gamma$ starting at $q$. By construction, this lift is necessarily a $\delta$-lift. Indeed, if $\sigma:[0, T] \rightarrow U$ is parameterized by arclength and $\widehat{\sigma}:\left[0, T^{\prime}\right] \rightarrow \Gamma$ satisfies $\Pi_{\Sigma}(\widehat{\sigma}(t))=\sigma(t)$ for some $0<T^{\prime} \leq T$, then

$$
\left|\frac{d}{d t} \operatorname{dist}^{\Omega}(\sigma(t), \widehat{\sigma}(t))\right| \leq C \operatorname{dist}^{\Omega}(\sigma(t), \widehat{\sigma}(t))
$$

and so

$$
\operatorname{dist}^{\Omega}(\sigma(t), \widehat{\sigma}(t)) \leq e^{C t} \operatorname{dist}^{\Omega}(p, q)<\epsilon \mu e^{C t}<\epsilon .
$$

Where we used that $t \leq T \leq l+d$ to obtain the final inequality. Furthermore, if $t<T$, then the lift $\widehat{\sigma}(t)$ may be extended past $t$ provided dist ${ }^{\Omega}(\sigma(t), \widehat{\sigma}(t))<\epsilon$. This proves that the leaf of a minimal disk closure has the simple lift property as claimed.

In the case of a minimal disk sequence, the argument is identical to the one above except that it uses the Harnack inequality to obtain the bound on the Lipschitz norms of straightening maps. We refer to Remark 3.6 and to [7, Appendix B] for the details on how to obtain this bound.

A surface with the simple lift property is one for which, in an effective sense, the universal cover of the surface can be properly embedded as a minimal disk near the surface. For this reason, to understand the topology of the surface, it is important to understand the lifting behavior of closed curves. With this in mind, we give the following definition.

Definition 4.3. Let $\Sigma \subset \Omega$ be an embedded minimal surface with the simple lift property. If $\gamma:[0,1] \rightarrow \Sigma$ is a piece-wise $C^{1}$ closed curve, then $\gamma$ has the open lift property if there exists a $\delta_{0}>0$ so that, for all $\delta_{0}>\delta>0, \gamma$ does not have a closed simple $\delta$-lift $\widehat{\gamma}:[0,1] \rightarrow \mathcal{N}_{\delta}(\Sigma)$. Otherwise, $\gamma$ has the closed lift property.

If a closed curve $\gamma$ has the closed lift property, then there is a sequence $\delta_{i} \rightarrow 0$ so that there are closed simple $\delta_{i}$-lifts $\widehat{\gamma}_{i}$ of $\gamma$. If it is possible to choose these lifts to be embedded we say $\gamma$ has the embedded closed lift property.

The next lemma says that if two loops satisfying certain geometric conditions have the open lift property, then their commutator has the closed lift property. Very roughly speaking, it does this by constructing an "effective" homomorphism from the space of loops in the leaf to $\mathbb{Z}$. Indeed, a curve $\widehat{\gamma}$ is a lift of $\gamma$ if and only if $\widehat{\gamma}^{-1}$ is a lift of $\gamma^{-1}$. Thus, $\gamma$ has the closed lift property if and only if $\gamma^{-1}$ does.

Proposition 4.4. Let $L \subset \Omega$ be an embedded minimal surface with the simple lift property and let

$$
\alpha:[0,1] \rightarrow L \text { and } \beta:[0,1] \rightarrow L
$$

be closed piece-wise $C^{1}$ curves satisfying the following properties:

(1) Both $\alpha$ and $\beta$ have the open lift property;

(2) $\alpha \cap \beta=\left\{p_{0}\right\}$ where $p_{0}=\alpha(0)=\beta(0)$;

(3) There exists a two sided pre-compact open set $U \subset L$ with $\alpha \cup \beta \subset U$. 
Then the curve $\mu=\alpha \circ \beta \circ \alpha^{-1} \circ \beta^{-1}$ has the closed lift property. If, in addition, both $\alpha$ and $\beta$ have the embedded lift property, then either $\mu$ has the embedded closed lift property, or one of the following two curves has the embedded closed lift property:

$$
\alpha \circ \beta \text { or } \beta \circ \alpha^{-1} \text {. }
$$

Proof. Consider a sequence $\delta_{i} \rightarrow 0$. As $\Sigma$ has the simple lift property, there exist constants $\epsilon_{i}$, embedded minimal disks $\Delta_{i}$ and simple $\delta_{i}$-lifts of $\mu$, $\widehat{\mu}_{i}$, so that $\Delta_{i} \cap \mathcal{N}_{\epsilon_{i}}(U)$ is a $\delta_{i}$-graph over $U, \widehat{\mu}_{i} \subset \Delta_{i}$ and the connected component $\Gamma_{i}$ of $\Delta_{i} \cap \mathcal{N}_{\epsilon_{i}}(U)$ containing $\mu_{i}$ is a $\delta_{i}$-cover of $U$. By reparameterizing appropriate restrictions of $\widehat{\mu}_{i}$, we obtain lifts of $\alpha, \alpha^{-1}, \beta$ and $\beta^{-1}$. We write $\widehat{\mu}_{i}=\widehat{\alpha}_{i} \circ \widehat{\beta}_{i} \circ \widehat{\alpha}_{i}^{-1} \circ \widehat{\beta}_{i}^{-1}$ where the $\widehat{\alpha}_{i}, \widehat{\beta}_{i}, \widehat{\alpha}_{i}^{-1}, \widehat{\beta}_{i}^{-1}:[0,1] \rightarrow \Gamma_{i}$ are lifts of the $\alpha, \beta, \alpha^{-1}, \beta^{-1}$.

Setting $p=\mu(0)$ we pick a small simply-connected neighborhood $V$ of $p$ that satisfies $V \subset U$. Because the $\Delta_{i}$ are embedded there is a natural way to order by height the components of $\Pi_{L}^{-1}(V) \cap \Delta_{i}$. We denote these ordered components by $\widehat{V}_{i}(1), \ldots, \widehat{V}_{i}\left(n_{i}\right)$. Let $\widehat{p}_{i}(0)=\widehat{\alpha}_{i}(0), \widehat{p}_{i}(1)=\widehat{\alpha}_{i}(1)=\widehat{\beta}_{i}(0), \widehat{p}_{i}(2)=\widehat{\beta}_{i}(1)=$ $\widehat{\alpha}_{i}^{-1}(0), \widehat{p}_{i}(3)=\widehat{\alpha}_{i}^{-1}(1)=\widehat{\beta}_{i}^{-1}(0), \widehat{p}_{i}(4)=\widehat{\beta}_{i}^{-1}(1)$ and notice that $\widehat{p}_{i}(j) \in$ $\widehat{V}_{i}(l(i, j))$ for some function $l$. Let

$$
\begin{aligned}
m_{i}[\alpha] & =l(i, 1)-l(i, 0), \\
m_{i}[\beta] & =l(i, 2)-l(i, 1), \\
m_{i}\left[\alpha^{-1}\right] & =l(i, 3)-l(i, 2), \text { and } \\
m_{i}\left[\beta^{-1}\right] & =l(i, 4)-l(i, 3),
\end{aligned}
$$

represent the (signed) number of sheets between the end points of $\widehat{\alpha}_{i}, \widehat{\beta}_{i}, \widehat{\alpha}_{i}^{-1}$ and $\widehat{\beta}_{i}^{-1}$. As both $\widehat{\alpha}_{i}$ and $\widehat{\beta}_{i}$ are open lifts these numbers are never zero. We now prove that $m_{i}[\alpha]=-m_{i}\left[\alpha^{-1}\right]$ and $m_{i}[\beta]=-m_{i}\left[\beta^{-1}\right]$ and, hence, $\hat{\mu}_{i}$ is closed. We consider two cases: $m_{i}[\alpha] m_{i}[\beta]>0$ and $m_{i}[\alpha] m_{i}[\beta]<0$.

In the first case we assume, with out loss of generality, that $m_{i}[\alpha], m_{i}[\beta]>0$. Using the fact that the $\Delta_{i}$ are embedded and that $U$ is two-sided, we see that there is a disjoint family of "parallel" lifts of $\alpha$ which we denote by $\widehat{\alpha}_{i}[j]$. The first member of the family is $\widehat{\alpha}_{i}[0]=\widehat{\alpha}_{i}$ and the subsequent members of the family are the lifts, $\widehat{\alpha}_{i}[k]$ of $\alpha$ which satisfy $\widehat{\alpha}_{i}[k](0)=l(i, 0)+k$. Namely, the lift $\widehat{\alpha}_{i}[k]$ starts $k$ sheets above $\widehat{\alpha}_{i}(0)$. By the embeddedness of $\Delta_{i}$ and the two-sidedness of $U$, the signed number of graphs between $\widehat{\alpha}_{i}[0](t)$ and $\widehat{\alpha}_{i}[k](t)$ is constant in $t$. Hence, $\widehat{\alpha}_{i}[k](1)=l(i, 1)+k$ - that is, the lifts have endpoint $k$ sheets above the endpoint of $\widehat{\alpha}_{i}$. Clearly, the $\widehat{\alpha}_{i}[k]$ are well-defined as long as $k \leq m_{i}[\beta]$. Furthermore, $\widehat{\alpha}_{i}\left[m_{i}[\beta]\right]$ has end point which is the same as the end point of $\widehat{\beta}_{i}$. Hence, since $\widehat{\alpha}_{i}\left[m_{i}[\beta]\right]^{-1}$ is a lift of $\alpha^{-1}$ starting at $\widehat{\beta}_{i}(1)$, the lift $\widehat{\alpha}_{i}\left[m_{i}[\beta]\right]^{-1}$ must be $\widehat{\alpha}_{i}^{-1}$. That is, $m_{i}[\alpha]=-m_{i}\left[\alpha^{-1}\right]$. An identical argument shows that $m_{i}[\beta]=-m_{i}\left[\beta^{-1}\right]$.

In the second case, we may suppose with out loss of generality that $m_{i}[\alpha]>0$ and $m_{i}[\beta]<0$. We first assume that $m_{i}[\alpha]+m_{i}[\beta]+m_{i}\left[\alpha^{-1}\right] \geq 0$ and obtain 
a contradiction. Under this hypothesis the end point of $\widehat{\alpha}_{i}^{-1}$ is not below that of the initial point of $\widehat{\alpha}_{i}$. As in the preceding argument, we can construct a family of "parallel" lifts of $\alpha^{-1}$. The first member of this family is $\widehat{\alpha}_{i}^{-1}$, the second lift in the family starts just below $\widehat{\alpha}_{i}^{-1}$ and the last lift in such family, $\widehat{\alpha}_{i}^{\prime}$ has end point that is the initial point of $\widehat{\alpha}_{i}$. As before, the number of graphs between the start and end points of a lift in this family is constant equal to $m_{i}\left[\alpha^{-1}\right]$. Since $\widehat{\alpha}_{i}^{\prime}$ has end point that is the initial point of $\widehat{\alpha}_{i}$, the lift $\left(\widehat{\alpha}_{i}^{\prime}\right)^{-1}$ must be $\widehat{\alpha}_{i}$. This implies that $m_{i}[\alpha]=-m_{i}\left[\alpha^{-1}\right]$. Since $m_{i}[\beta]<0$, this leads to a contradiction. Hence, $m_{i}[\alpha]+m_{i}[\beta]+m_{i}\left[\alpha^{-1}\right]<0$. Again one constructs a family of parallel lifts starting from $\widehat{\alpha}_{i}$ and ending with a curve with initial point the end point of $\widehat{\alpha}_{i}^{-1}$ which again implies that $m_{i}[\alpha]=-m_{i}\left[\alpha^{-1}\right]$. The same argument shows that in this case $m_{i}[\beta]=-m_{i}\left[\beta^{-1}\right]$.

Finally, we note that if $\alpha$ and $\beta$ have the embedded lift property, then, because they meet at only one point, the curves $\widehat{\alpha}_{i} \circ \widehat{\beta}_{i}, \widehat{\beta}_{i} \circ \widehat{\alpha}_{i}^{-1}$ and $\widehat{\alpha}_{i}^{-1} \circ \widehat{\beta}_{i}^{-1}$ are all embedded. Hence, the only way that $\widehat{\mu}_{i}$ can fail to be embedded is if one of the the first two is closed.

\section{MAIN PROOF}

Rather than prove Theorems 1.1 and 1.2 directly we prove slightly more general results. To do so we will restrict the geometry of the three-manifolds $\Omega$ we consider.

Assumption 5.1. Let $\Omega$ be the interior of a complete, oriented, three-manifold with (possibly empty) boundary $N=\bar{\Omega}$ satisfying the following properties:

(1) The boundary of $N$ is mean-convex;

(2) There is an exhaustion $\left\{\Omega_{t}\right\}_{t \in[0,1)}$ of $\Omega$ so that each $\Omega_{t}$ is pre-compact in $N, \partial \Omega_{t}$ is mean-convex and $\left\{\partial \Omega_{t}\right\}_{t \in(0,1)}$ foliates $\Omega \backslash \bar{\Omega}_{0}$;

(3) $\Omega$ contains no closed minimal surfaces.

Note that $N$ does not have to be compact. For instance, $\mathbb{H}^{3}$ satisfies Assumption 5.1. Minimal surfaces in such an $\Omega$ automatically satisfy a certain type of uniform isoperimetric inequality. This follows immediately from work of $\mathrm{B}$. White [24].

Lemma 5.2. If $\Omega$ satisfies the conditions of Assumption 5.1 $U \subset \Omega$ is a precompact subset of $\Omega$ and $\Sigma$ is a compact minimal surface with boundary, $\partial \Sigma \subset U$, then

(1) There is a pre-compact open set $U^{\prime}$ depending only on $U$ so that $\Sigma \subset U^{\prime}$;

(2) There is an increasing function, $\Psi_{U}: \mathbb{R}^{\geq 0} \rightarrow \mathbb{R}^{\geq 0}$, depending only on $U$ and satisfying $\Psi_{U}(0)=0$ and

$$
|\Sigma| \leq \Psi_{U}(|\partial \Sigma|)
$$

Proof. By Assumption 5.1, there is a pre-compact open subset $U^{\prime}=\Omega_{t_{0}}$ with $U \subset \Omega_{t_{0}}$. As $\Omega \backslash \Omega_{t_{0}}$ is foliated by mean-convex subsets, the strong maximum 
principle and the fact that $\partial \Sigma \subset \Omega_{t_{0}}$ implies that $\Sigma \subset \Omega_{t_{0}}$. Finally, the existence of the function $\Psi_{U}$ follows immediately from [24, Theorem 2.1] applied to $\overline{U^{\prime}}$.

Under Assumption 5.1 we have the following gluing property that allows us to "fill in" curves with the embedded closed-lift property.

Lemma 5.3. Suppose that $\Omega$ satisfies Assumption 5.1 If $L \subset \Omega$ is an embedded minimal surface with the simple lift property and $\gamma:[0,1] \rightarrow L$ has the embedded closed lift property, then there exists a smooth minimal surface $\Delta$ properly embedded in $\Omega \backslash \gamma$ so that:

(1) $\Delta$ has finite area and is contained in a compact subset of $\Omega$;

(2) $\gamma=\bar{\Delta} \backslash \Delta$ and $\Delta \cup \gamma$ is connected;

(3) $\Delta \cap L$ is a non-empty open and closed subset of $L \backslash \gamma$; and

(4) If $\gamma$ is embedded, then $\Delta$ is a disk.

Proof. By hypothesis, there exists a sequence of closed embedded simple $\frac{1}{n}$-lifts $\gamma_{n}$ of $\gamma$. Hence, each $\gamma_{n}$ bounds a minimal disk $\Delta_{n}^{\prime}$ inside of the minimal disks $\Delta_{n}$. Clearly, there is a fixed pre-compact subset $U$ of $\Omega$ containing $\gamma$ together with all of the $\gamma_{n}$. Furthermore, the length of each $\gamma_{n}$ is bounded by twice the length of $\gamma$ and so there is a uniform bound on $\left|\partial \Delta_{n}^{\prime}\right|$. As a consequence, Lemma 5.2 implies that there is a precompact subset $U^{\prime}$ of $\Omega$ so that the sequence of disks $\Delta_{n}^{\prime}$ are contained in $U^{\prime}$ and, moreover, have uniformly bounded area. A result of Schoen and Simon [19] - see Theorem B.1 for the statement - then gives uniform curvature bounds for the $\Delta_{n}^{\prime}$ on compact subsets of $\Omega \backslash \gamma_{n}$. Hence, up to passing to a subsequence, Theorem 3.5 and the area bounds imply that the $\Delta_{n}^{\prime}$ converge in $C_{\text {loc }}^{\infty}(\Omega \backslash \gamma)$ to a properly embedded minimal surface $\Delta \subset \Omega \backslash \gamma$ of finite area and bounded curvature on compact sets of $\Omega \backslash \gamma$. As each $\Delta_{n}^{\prime}$ is contained in $U^{\prime}$, it follows that $\Delta \subset \bar{U}^{\prime}$. This proves Items (1). Item (2) follows from the set theoretic convergence of $\bar{\Delta}_{n}^{\prime}$.

As the $\Delta_{n}$ are contained in $\frac{1}{n}$ graphs over a fixed neighborhood $V$ of $\gamma, \Delta \backslash \gamma$ contains a component of $V \backslash \gamma$ and so $\Delta \backslash \gamma$ is non-empty and is contained in $L$. Indeed, the nature of the convergence implies that $\Delta \backslash \gamma$ is an open and closed subset of $L \backslash \gamma$.

If $\gamma$ is embedded, then the $\widehat{\gamma}_{n}$ converge to $\gamma$ with multiplicity one. Clearly, to prove Item (4) it suffices to show that the $\Delta_{n}^{\prime}$ also converge to $\Delta$ with multiplicity one. This is most conveniently done using the language of varifolds - we refer to [20] for details.

Let $V_{n}$ be the integer multiplicity rectifiable varifold associated to $\bar{\Delta}_{n}^{\prime}$. As $\gamma$ is piecewise smooth, the nature of the convergence of $\gamma_{n}$ toward $\gamma$ implies that $\bar{\Delta}_{n}^{\prime}$ has uniformly bounded first variation. Indeed, since $\Delta_{n}$ is minimal, the first variation measure of $V_{n}$ is supported along $\gamma_{n}$. As the $V_{n}$ also have uniformly bounded mass, we may apply the compactness theory for integer multiplicity rectifiable varifolds to see that (up to passing to a further subsequence) $V_{n}$ converges in the sense of varifolds (in $\Omega$ ) to an integer multiplicity rectifiable varifold $V$. It follows from the nature of the convergence that $\operatorname{spt} V=\bar{\Delta}$ and, moreover, at any point of $\Delta$ the multiplicity of $V$ is a positive integer. Moreover, the first variation measure of $V$ is 
supported along $\gamma$. Notice that as $L$ is a smooth minimal surface and $\operatorname{spt} V \subset L$, the constancy theorem implies that the multiplicity of each component of $\operatorname{spt} V \backslash \gamma$ is constant.

Fix a point $p \in \gamma$ and a small open neighborhood $W \subset L$ about $p$ choosen small enough so that $\gamma$ divides $W$ into exactly two components $W_{-}$and $W_{+}$. If both $W_{-}$and $W_{+}$meet spt $V$, then it follows from the strong unique continuation princple for smooth minimal surfaces that $\operatorname{spt} V$ is a closed minimal surface in $\Omega$. This violates Assumption 5.1 and so - up to relabelling - we may assume that $W_{-} \cap \operatorname{spt} V=\emptyset$. As $\gamma_{n}$ converge to $\gamma$ with multiplicity one, the nature of the convergence of $\Delta_{n}^{\prime}$ to $\Delta$ then immediately implies that the multiplicity of $V$ is one which proves the claim.

Corollary 5.4. Suppose $\Omega$ satisfies Assumption 5.1 If $L \subset \Omega$ is an embedded minimal surface with the simple lift property and $\gamma$ is a closed embedded curve in $L$ with the closed lift property, then $\gamma$ is separating.

Proof. Let $\Delta$ be the surface given by Lemma 5.3. If $\gamma$ is non-separating, then $L \backslash \gamma$ is connected and so, by the previous lemma, $L \backslash \gamma \subset \Delta$. Therefore, by strong unique continuation for smooth minimal surfaces, $\bar{\Delta}=\Delta \cup \gamma=\Delta \cup L$ is a closed minimal surfaces of finite area in $\Omega$ which contradicts Assumption 5.1

We are now in a position to prove that surfaces with the simple lift property in regions satisfying Assumption 5.1 must have genus zero.

Proposition 5.5. Suppose $\Omega$ satisfies Assumption 5.1 If $L \subset \Omega$ is an embedded minimal surface with the simple lift property in $\Omega$, then $L$ has genus zero.

Proof. Arguing by contradiction, suppose that $L$ has genus greater than zero. Then, by the classification of surfaces, there exist two piece-wise smooth, non-separating Jordan curves $\alpha, \beta:[0,1] \rightarrow L$ and a two-sided pre-compact set $U \subset L$ such that the following holds:

- $\alpha \cap \beta=p=\alpha(0)=\beta(0)=\alpha(1)=\beta(1)$;

- $L \backslash(\alpha \cup \beta)$ is connected;

- $\alpha \cup \beta \subset U$.

By Lemma 5.4 both curves have the open lift property and so $\alpha$ and $\beta$ satisfy the hypothesis of Proposition 4.4 Hence, there exists a sequence of closed curves $\nu_{n}$ that are closed simple $\frac{1}{n}$-lifts of $\nu=\alpha \circ \beta \circ \alpha^{-1} \circ \beta^{-1}$. Proposition 4.4 further tells us that either the curve $\mu=\nu$, the curve $\mu=\alpha \circ \beta$ or the the curve $\mu=\beta \circ \alpha^{-1}$ has the embedded closed lift property. In all cases, Lemma 5.3 gives a minimal surface $\Delta$ properly embedded in $\Omega \backslash \mu$ and that $[\Delta \backslash \mu] \cap L$ is a non-empty, open and closed subset of $L \backslash \mu$. As $L \backslash \mu$ is connected, $\Delta=L \backslash \mu$. Hence, by the strong unique continuation property of smooth minimal surfaces, $\bar{\Delta}=\Delta \cup L$ is a properly embedded minimal surface in $\Omega$ of finite area and we contradict Assumption 5.1

Clearly, Theorem 1.2 follows from Proposition 4.2 Indeed, the region $\Omega$ of Theorem 1.2 can be seen to satisfy Assumption 5.1 by taking the exhaustion to be $\Omega$ itself. 


\section{Minimal Disk Sequences}

In this section we conclude the proof of Theorem 1.1 We first show that the leaves of the limit lamination do not have many holes.

Proposition 6.1. If $(\Omega, K, \mathcal{L}, \mathcal{S})$ is a minimal disk sequence and $L$ is a leaf of $\mathcal{L}$, then

(1) If $L$ is two-sided, then $L$ is either a disk or an annulus;

(2) If $L$ is one-sided, then $L$ is a Möbius band.

Proof. We will argue by contradiction. For any three separating Jordan curves $\gamma_{1}$, $\gamma_{2}$ and $\gamma_{3}$ in $L$ with the property that no one of the curves separates the other two it is the case that $L \backslash\left(\gamma_{1} \cup \gamma_{2} \cup \gamma_{3}\right)$ has four components $L_{1}, L_{2}, L_{3}, L_{4}$. Label the $L_{i}$ so that $\bar{L}_{1} \cap\left(\gamma_{2} \cup \gamma_{3}\right)=\emptyset, \bar{L}_{2} \cap\left(\gamma_{1} \cup \gamma_{3}\right)=\emptyset, \bar{L}_{3} \cap\left(\gamma_{1} \cup \gamma_{2}\right)=\emptyset$ and $\gamma_{1} \cup \gamma_{2} \cup \gamma_{3} \subset \bar{L}_{4}$. By Proposition 5.5, $L$ has genus zero. Hence, if $L$ is neither a disk, an annulus nor a Möbius band, then the classification of surfaces implies that the $\gamma_{i}$ may be choosen so that

- $L_{1}, L_{2}$ and $L_{3}$ are not disks;

- $L_{4}$ is two-sided.

[THIS PART NEEDS TO BE CHANGED] We claim that for such a choice, $\gamma_{1}, \gamma_{2}$ and $\gamma_{3}$ have the open lift and embedded lift property. Indeed, being embedded curves, they clearly have the embedded lift property. Suppose $\gamma_{i}$ had the closed lift property, then applying Lemma 5.3 would give that $\gamma_{i}$ is the boundary of a disk $\Delta \subset L$ contradicting our choice of $\gamma_{i}$. [!!!]

Let $\sigma$ be an embedded arc in $L_{4}$ which connects $\gamma_{1}(0)$ to $\gamma_{2}(0)$. Notice that the classification of surfaces tells us that such $\sigma$ exists and does not separate $L_{4}$. Consider the new closed curve $\gamma_{4}=\sigma^{-1} \circ \gamma_{2} \circ \sigma$. By an argument analogous to the one described before, this curve must also have the open lift property. In fact, the embeddedness of $\sigma$ and of $\gamma_{2}$ and the fact that $\gamma_{2}$ has the open-lift property imply that $\gamma_{4}$ has the embedded-lift property.

We now consider the closed curve $\nu=\gamma_{1} \circ \gamma_{4} \circ \gamma_{1}^{-1} \circ \gamma_{4}^{-1}$. Proposition 4.4 implies that either the curve $\mu=\nu$, the curve $\mu=\gamma_{1} \circ \gamma_{4}$ does or the curve $\mu=\gamma_{4} \circ \gamma_{1}^{-1}$ has the embedded closed lift property. In all cases, let $\Delta$ be the embedded minimal surface given by Lemma 5.3. The fact that $\Delta$ is connected and that $\gamma_{1} \cup \gamma_{2} \subset \bar{\Delta}$ together imply that $L_{4} \subset \Delta$. However, as $\gamma_{3}$ is a Jordan curve disjoint from $\nu, \gamma_{3}$ must be the limit of embedded closed curves in $\Delta_{n}$ - that is, it has the embedded closed lift property. This is contradiction and proves the Proposition.

We next show that in the case of minimal disk sequences, the leaves of the limit lamination are two-sided.

Proposition 6.2. Let $\Omega$ satisfy Assumption 5.1 If $(\Omega, K, \mathcal{L}, \mathcal{S})$ is a minimal disk sequence and $L$ is a leaf of $\mathcal{L}$, then $L$ is two-sided.

Proof. Suppose that $L$ is a one-sided leaf of $\mathcal{L}$. By Proposition 6.1 $L$ is a Möbius band. As a consequence, there is a closed Jordan curve $\gamma:[0,1] \rightarrow L$ that is 
non-separating and so, by Lemma 5.4, has the open lift property. Let $U$ be an open pre-compact neighborhood of $\gamma$ and pick $\epsilon>0$ so that $\overline{\mathcal{N}}_{\epsilon}(U)$ is a regular neighborhood. As $\gamma$ is non-separating, $U$ is one-sided and, indeed, the surface $M=\Pi_{L}^{-1}(\gamma) \cap \overline{\mathcal{N}}_{\epsilon}(U)$ is a closed Möbius band.

Let $\Sigma_{i}$ be the surfaces in $\mathcal{S}$. There are curves, $\widehat{\gamma}_{i}$, which are components of $\Sigma_{i} \cap M$ containing $\delta$-lifts of $\gamma$ for any $\delta$ sufficiently small. In particular, the $\widehat{\gamma}_{i}$ are proper, but not closed, in $M$. Furthermore, after possibly shrinking $\epsilon$, they are monotone in the sense that $\left(\widehat{\gamma}_{i}^{\prime}\right)^{\top} \neq 0$ and $\widehat{\gamma}_{i}$ meets $\partial M$ transversely. Finally, for $i$ large enough, the map $\Pi_{L}: \widehat{\gamma}_{i} \rightarrow \gamma$ contains a three-fold cover. We claim this yields a contradiction.

To see this consider $\pi: \widetilde{M} \rightarrow M$ the oriented double cover of $M$. As $\widetilde{M}$ is an annulus and $\widehat{\gamma}_{i}$ is monotone:

- $\widetilde{M}=\mathbb{S}^{1} \times[-1,1]$ with coordinates $(\theta, z)$;

- $M=\widetilde{M} / \sim$ with $(\theta, z) \sim(\theta+\pi,-z)$;

- $\mathbb{S}^{1} \times\{0\}=\pi^{-1}(\gamma)$;

- $\widetilde{\gamma}_{i}=\pi^{-1}\left(\widehat{\gamma}_{i}\right)$ is a graph over $\mathbb{S}^{1}$.

As $\widetilde{\gamma}_{i}$ is a graph, we may parametrize $\widetilde{\gamma}_{i}(\theta)$ as $\left(\theta, v_{i}(\theta)\right)$ for $\theta \in\left[0, T_{i}\right]$ and some continuous function $v_{i}$ with $\left|v_{i}(0)\right|=\left|v_{i}\left(T_{i}\right)\right|=1$ and $\left|v_{i}(\theta)\right|<1$ for $\theta \in\left(0, T_{i}\right)$. Since $\widehat{\gamma}_{i}$ contains a three-fold cover of $\gamma, T_{i}>3 \pi$. The embeddedness of $\widehat{\gamma}_{i}$ implies that for any $\theta \in\left[0, T_{i}-\pi\right], v_{i}(\theta+\pi) \neq-v_{i}(\theta)$ and for any $\theta \in\left[0, T_{i}-2 \pi\right], v_{i}(\theta+$ $2 \pi) \neq v_{i}(\theta)$. Without loss of generality, we assume that $v_{i}(0)=-1$. Consider the continuous function $g_{i}$ defined for $\theta \in\left[0, T_{i}-2 \pi\right]$ by $g_{i}(\theta)=v_{i}(\theta+2 \pi)-v_{i}(\theta)$. Notice that $g_{i}\left(T_{i}-2 \pi\right)<0$ if and only if $v_{i}\left(T_{i}\right)=-1$. Hence, as $g_{i}(0)>$ 0 , the intermediate value theorem implies that $v_{i}\left(T_{i}\right)=1$. Finally, consider the continuous function $f_{i}$ defined for $\theta \in\left[0, T_{i}-\pi\right]$ by $f_{i}(\theta)=v_{i}(\theta+\pi)+v_{i}(\theta)$. Clearly, $f_{i}(0)<0$ and $f_{i}\left(T_{i}-\pi\right)>0$. Hence the intermediate value theorem contradicts the fact that $f_{i}(\theta) \neq 0$; completing the proof.

We now finish the proof of Theorem 1.1 For completeness, we recall its statement.

Theorem 1.1. Let $\Omega$ be the interior of a compact oriented three-manifold $N=$ $\bar{\Omega}$ with mean-convex boundary. If $\Omega$ contains no closed minimal surfaces and $(\Omega, K, \mathcal{L}, \mathcal{S})$ is a minimal disk sequence, then the leaves of $\mathcal{L}$ are either disks or annuli. Furthermore, if $L$ is a leaf of $\mathcal{L}$ with the property that $\bar{L}$ - the closure in $\Omega$ of $L-i$ is a properly embedded minimal surface, then $\bar{L}$ is either a disk or it is an annulus which is disjoint from $K$.

Proof. We first note that $\Omega$ satisfies the conditions of Assumption 5.1 by taking the exhaustion to be $\Omega$ itself. Moreover, each leaf of $\mathcal{L}$ has the simple lift property by Proposition 4.2 Hence, Propositions 6.1 and 6.2 together imply $L$ is either a disk or an annulus. The remainder of the theorem follows from the deeper result of Colding and Minicozzi that we summarized in Proposition 3.8 Indeed, if $L$ is a leaf of $\mathcal{L}$ with $\bar{L}$ a properly embedded minimal surface, then it is regular at each $p \in \bar{L} \cap K$. Hence, by Proposition 3.8, $\bar{L} \cap K$ is a discrete set of points in $\bar{L}$. As $L$ is either a disk or an annulus, if $\bar{L}$ is an annulus it must be disjoint from $K$. 


\section{APPENDIX A. EXAMPLES}

A.1. One-sided Limit Leaf. In this section, we construct a simply-connected minimal surface $M$ embedded in a solid torus that is not properly embedded. Moreover, its closure is a lamination in the solid torus consisting of three leaves. The leaf $M$ and two limit leaves. One limit leaf is an annulus while the other is a Möbius band.

Let $T$ be a solid torus obtained by revolving a disk $\mathcal{D}$ in the $\left(x_{1}, x_{3}\right)$-plane around the $x_{3}$-axis. We take $\mathcal{D}$ small enough so that $T$ is mean-convex and there exists a stable minimal Möbius band $M$ embedded in $T$ whose double cover is also stable and with boundary a simple closed curve in $\partial T$. For the existence of such a surface we refer to [17]. Since the double cover of $M$ is stable, a normal neighbourhood of $M$ can be foliated by minimal surfaces and, except for $M$ itself, the leaves of this foliation are two-sided annuli. Let $\Sigma$ denote the outermost leaf of this foliation and let $W$ denote the open region between $M$ and $\Sigma$. Let $\Sigma_{t}$, $t \in[0.1]$, be an indexing of the leaves of the foliation with $\Sigma_{0}=M$ and $\Sigma_{1}=\Sigma$. Let $\widetilde{T}$ be the universal cover of $T$ with the induced metric. We realize $\widetilde{T}$ as

$$
\widetilde{T}=\left\{(x, y, z): x^{2}+y^{2} \leq 1\right\} \subset \mathbb{R}^{3}
$$

in a manner so that for any $\alpha \in \mathbb{R}$ the map

$$
G_{\alpha}: \widetilde{T} \rightarrow \widetilde{T}, \quad G(x, y, z)=(x, y, z+\alpha)
$$

is an isometry and for any $t \in \mathbb{R}$ the set

$$
B_{t}:=\{(x, y, z) \in \widetilde{T} \mid z=t\}
$$

is a minimal surface that is a lift of a disk obtained by intersecting $T$ with a vertical plane containing the $z$-axis. The maps $G_{2 \pi n}, n \in \mathbb{Z}$ are the deck transforms of $\widetilde{T}$. Let $\Pi: \widetilde{T} \rightarrow T$ denote the natural projection. Given an embedded surface $S \in \widetilde{T}$, if for any $n \in \mathbb{Z} \backslash\{0\}$ it holds that $G_{2 \pi n}(S) \cap S=\emptyset$, then $\Pi(S)$ is embedded in $T$.

The Möbius band $M$ lifts to a strip $\widetilde{M}$ with boundary consisting of two curves in $\partial \widetilde{T}$ and, being a lift, is invariant by the deck transforms $G_{2 \pi n}, n \in \mathbb{Z}$. The strip $\widetilde{M}$ is two-sided and separates $\widetilde{T}$ into two components. Each leaf $\Sigma_{t}$ of the foliation, $t \in(0,1]$, lifts to two strips $\widetilde{\Sigma}_{t}^{+}$and $\widetilde{\Sigma}_{t}^{-}$on opposite sides of $\widetilde{M}$ and this gives a foliation of a two-sided normal neighbourhood of $\widetilde{M}$. We shall denote by $\widetilde{W}$ the region foliated by the leaves $\widetilde{\Sigma}_{t}^{+}$and denote such leaves by $\widetilde{\Sigma}_{t}$. Given a point $p \in \widetilde{W}$, then $p \in \widetilde{\Sigma}_{t}$ for a certain $t \in(0,1)$ and we denote that $t$ by $t(p)$. Note that $\partial \widetilde{W} \cap \partial \widetilde{T}$ consists of two disconnected components, $\Delta_{1}$ and $\Delta_{2}$, and let $\alpha_{i} \subset \Delta_{i}$, $i=1,2$ be analytic curves such that the following holds:

- $\alpha_{i}$ intersects $\partial \widetilde{\Sigma}_{t}, t \in(0,1)$, in exactly one point;

- $\alpha_{i}$ converges to $\partial \widetilde{\Sigma}_{1} \cap \Delta_{i}$ as $z$ goes to infinity and to $\partial \widetilde{M} \cap \Delta_{i}$ as $z$ goes to minus infinity.

Let $\widetilde{W}_{n}$ be the region in $\widetilde{W}$ in between the minimal disks $B_{ \pm 2 \pi n}$. Then, $\widetilde{W}=$ $\bigcup_{n} \widetilde{W}_{n}$ and $\partial \widetilde{W}_{n}$ consists of six surfaces: four minimal surfaces, $\widetilde{\Sigma}_{0}^{n}=\widetilde{M} \cap \widetilde{W}_{n}$, $\widetilde{\Sigma}_{1}^{n}=\widetilde{\Sigma}_{1} \cap \widetilde{W}_{n}, B_{+}^{n}=B_{2 \pi n} \cap \widetilde{W}_{n}$, and $B_{-}^{n}=B_{-2 \pi n} \cap \widetilde{W}_{n}$ and two mean convex 
surfaces $\Delta_{1}^{n}=\Delta_{1} \cap \widetilde{W}_{n}$ and $\Delta_{2}^{n}=\Delta_{2} \cap \widetilde{W}_{n}$. Since the contact angle between such surfaces is less than $\pi$, the boundary of $\widetilde{W}_{n}$ is mean-convex and a good barrier to solve Plateau problem.

Let $\gamma_{n} \in \partial W_{n}$ be a piece-wise smooth simple closed curve constructed in the following way. The curve $\gamma_{n}$ is given by the union $\alpha_{1}^{n} \cup \beta_{+}^{n} \cup \alpha_{2}^{n} \cup \beta_{-}^{n}$ where $\alpha_{i}^{n}=\alpha_{i} \cap \widetilde{W}_{n}$. The curve $\beta_{+}^{n}$ is an arc connecting the endpoints, $p_{i}^{n}$ of $\alpha_{i}^{n}$ in $B_{+}^{n}$. If $t_{n}=t\left(p_{1}^{n}\right)=t\left(p_{2}^{n}\right)$, then we take $\beta_{+}^{n}$ to lie in $\Sigma_{t_{n}}$. Otherwise, we take $\beta_{+}^{n}$ to intersect each $\Sigma_{t}$ in at most one point. We choose the the curve $\beta_{-}^{n}$ in an analogous manner in $B_{-}^{n}$. Clearly, by our choices of $\alpha_{i}$, for $n$ sufficiently large,

$$
\max _{p \in \beta_{+}^{n}}\{t(p)\}<\min _{p \in \beta_{-}^{n}}\{t(p)\} .
$$

This implies that for any $n$ sufficiently large and any $m \in \mathbb{Z} \backslash\{0\}$ then

$$
G_{2 \pi m}\left(\gamma_{n}\right) \cap \gamma_{n}=\emptyset
$$

By a result in Meeks and Yau [18], $\gamma_{n}$ is the boundary of an embedded, area minimizing disk $D_{n} \subset \widetilde{W}_{n}$. Since it is area minimizing and, for $n$ large, $\partial G_{2 \pi m}\left(D_{n}\right) \cap$ $\gamma_{n}=\emptyset$ for any $m \in \mathbb{Z} \backslash\{0\}$, it follows that

$$
G_{2 \pi m}\left(D_{n}\right) \cap D_{n}=\emptyset,
$$

giving that $\Pi\left(D_{n}\right)$ is also embedded. Moreover, since $D_{n}$ is area minimizing and $\alpha_{i}^{n}, i=1,2$, are analytic curves, it satisfies curvature estimates up to $\alpha_{i}, i=1,2$ and a standard compactness argument gives that it converges to a complete simplyconnected minimal surface $D_{\infty}$ embedded in $\widetilde{T}$ with boundary $\alpha_{i}, i=1,2$. By construction,

$$
G_{2 \pi m}(D) \cap D=\emptyset
$$

for any $m \in \mathbb{Z} \backslash\{0\}$, therefore, if we let $D=\Pi\left(D_{\infty}\right)$, then $D$ is a complete embedded disk. Clearly it is not properly embedded $T$. By curvature estimates for stable minimal surfaces, $\bar{D}$, the closure of $D$ in $T$, is a minimal lamination. We claim that $\bar{D}$ consists of three leaves, $D$ itself and two limit leaves $D_{1}$ and $D_{2}$. By construction, $\bar{D}$ contains a compact leaf $D_{1}$ with boundary $\partial \Sigma$ and a compact leaf $D_{2}$ with boundary $\partial M$. Using the foliation $\Sigma_{t}$ and the strong maximum principle one concludes that $D_{1}=\Sigma$ and $D_{2}=M$.

A.2. Torus limit leaf. In this section we construct a three-manifold, $\Omega$, and a complete, embedded disk $\Delta \subset \Omega$ whose closure, $\bar{\Delta}$, is a minimal lamination in $\Omega$ one of whose leaves is a minimal torus. More specifically, we take $\Omega=\mathbb{T}^{2} \times \mathbb{R}$ together with a certain metric for which Assumption 5.1 does not hold. In $\Omega$ we construct an embedded minimal disk $\Delta$ that is not properly embedded and so the closure of $\Delta$ is a proper minimal lamination in $\Omega$ consisting of five leaves. The leaf $L_{1}=\Delta$ and four limit leaves. Two of the limit leaves are the tori $L_{2}=\mathbb{T}^{2} \times\{-1\}$ and $L_{3}=\mathbb{T}^{2} \times\{1\}$, the other two $L_{4}$ and $L_{5}$ are non-proper annuli with $\bar{L}_{4}=$ $\bar{L}_{5}=L_{2} \cup L_{3}$. The original idea for this construction is due to D. Hoffman; we refer also to [3] for a related construction. 
We begin by constructing a metric $g$ on the cylinders

$$
C=\mathbb{S}_{\theta}^{1} \times \mathbb{R}_{t}
$$

Consider the metric

$$
g_{0}=(2+\cos \pi t) d \theta^{2}+d t^{2}
$$

and the foliation of $C$ by circles, $\mathbb{S}^{1}[t]=\mathbb{S}^{1} \times\{t\} \subset C$. It is an elementary computation to see that these circles all have constant curvature. Moreover, the leaves which are geodesics are $\mathbb{S}^{1}[i]$ for $i \in \mathbb{Z}$. When $i \in 2 \mathbb{Z}$ these geodesics are unstable while for $i \in 2 \mathbb{Z}+1$ they are stable. Let $U$ be the connected component of $C \backslash\left(\mathbb{S}^{1}[-1] \cup \mathbb{S}^{1}[1]\right)$ which contains $(0,0)$. Similarly, we consider the foliation $\alpha_{\theta}=(\{\theta\} \times \mathbb{R}) \cap U$ of $U$. It is clear that all the leaves of this foliation are geodesics. Finally, let us denote by $T_{v}$ the "translation" map $T_{v}((\theta, t))=(\theta+v, t)$ which is clearly an isometry and by $R$ the isometric involution given by $R((\theta, t))=$ $(-\theta,-t)$.

Standard methods - e.g., a shooting method or a minimization procedure in the universal cover of $C$ - produce an embedded geodesic $\gamma_{+}:[0, \infty)_{s} \rightarrow \gamma_{+} \subset U$ with $\gamma_{+}(0)=(0,0)$ and so that the $t$ coordinate of $\gamma_{+}(s)$ is monotonically increasing in $s$. Here $s$ is the arclength parameter. It is clear that $\gamma_{+}$must accumulate at $\mathbb{S}^{1}[1]$. Let $\gamma=\gamma_{+} \cup R\left(\gamma_{+}\right)$. This is a non-proper geodesic in $C$ which accumulates at $\mathbb{S}^{1}[-1] \cup \mathbb{S}^{1}[1]$. It follows also from the construction that if $\gamma_{v}:=T_{v}(\gamma)$ then $\left\{\gamma_{v}\right\}_{v \in \mathbb{R}}$ is a foliation of $U$. With that in mind, let $\gamma_{-}=\gamma_{-\pi / 2}$ and $\gamma_{+}=\gamma_{\pi / 2}$ and let $V$ be the component of $U \backslash\left(\gamma_{-} \cup \gamma_{+}\right)$which contains $(0,0)$.

We now modify the metric $g_{0}$, so that geodesics which pass through $(0,0)$ are unstable. To that end, pick a compactly supported function $\phi \in C_{0}^{\infty}(V)$ so that

- $0 \leq \phi \leq 1$

- $\operatorname{spt}(\phi) \subset B_{2 \epsilon} \subset V \cap\left(-\frac{\pi}{2}, \frac{\pi}{2}\right) \times(-1,1)$;

- $\phi \circ R=\phi$;

- $\phi=1$ on $B_{\epsilon}$.

Here $B_{r}$ is the geodesic ball (with respect to $\left.g_{0}\right)$ about $(0,0)$ of radius $r$ and we choose $\phi$ so that $2 \epsilon$ is smaller than the injectivity radius of $g_{0}$ at $(0,0)$. Now fix a point $p \in \mathbb{S}^{2}$ and let $g_{\mathbb{S}^{2}}$ be the round metric of curvature one on $\mathbb{S}^{2}$. We denote by $\mathcal{B}_{r}$ the geodesic ball of radius $r$ in $\mathbb{S}^{2}$ about $p$. As $B_{2 \epsilon}$ and $\mathcal{B}_{\frac{7}{8} \pi}$ are disks, there is a smooth diffeomorphism

$$
\Psi: B_{2 \epsilon} \rightarrow \mathcal{B}_{\frac{7}{8} \pi}
$$

Moreover, we may choose this smooth diffeomorphism so that

- $\Psi((0,0))=p$

- $\Psi\left(B_{\epsilon}\right)=\mathcal{B}_{\frac{3}{4} \pi}$

- $\Psi \circ R=\widetilde{R} \circ \Psi$ - here $\widetilde{R}$ is the isometry of $\mathbb{S}^{2}$ given by rotating $180^{\circ}$ around the line through $p$ and $-p$.

We now set

$$
g_{1}=(1-\phi) g_{0}+\phi \Psi^{*} g_{\mathbb{S}^{2}}
$$

Geodesics of $g_{1}$ that pass through $(0,0)$ are, by construction, unstable. 
Lemma A.1. If $\gamma$ is a geodesic in $V$ for $g_{1}$ such that $(0,0) \in \gamma$ and $B_{\epsilon} \cap \gamma$ is proper in $B_{\epsilon}$, then $\gamma$ is unstable.

Proof. If $\gamma$ is such a geodesic, then $\Psi\left(\gamma \cap B_{\epsilon}\right)$ is a proper geodesic in $\mathcal{B}_{\frac{3}{4} \pi}$ that contains $p$. Hence $\Psi\left(\gamma \cap B_{\epsilon}\right)$, has length at least $\frac{3}{2} \pi>\pi$ and so is unstable.

Note that the curves $\mathbb{S}_{ \pm 1}^{1}, \gamma_{ \pm}$and $\alpha_{\pi}$ all remain geodesics for $g_{1}$ as $g_{1}=g_{0}$ in a neighborhood of these curves. Furthermore, as $\phi \circ R=\phi$ and $\Psi \circ R=\tilde{R} \circ \Psi, R$ is also an isometry of $g_{1}$.

Our goal now is to construct the desired disk $\Delta$. To that end, let $\Omega=\mathbb{S}_{\psi}^{1} \times C=$ $\mathbb{T}^{2} \times \mathbb{R}$ have the product metric

$$
g_{\Omega}=d \psi^{2}+g_{1} .
$$

Set $U^{\prime}=\mathbb{S}^{1} \times U$ and $V^{\prime}=\mathbb{S}^{1} \times V$ and note that $\Gamma_{ \pm}=\mathbb{S}^{1} \times \gamma_{ \pm}$are totally geodesic cylinders which accumulate at the totally geodesic tori $\mathbb{T}^{2}[ \pm 1]=\mathbb{S}^{1} \times$ $\mathbb{S}^{1}[ \pm 1]$. Likewise, let $A=\mathbb{S}^{1} \times \alpha_{\pi} \subset U^{\prime}$, which is a totally geodesic annulus. The following "translation" map is an isometry of $g_{\Omega}$ for $v \in \mathbb{R}$

$$
T_{v}((\psi, \theta, t))=(\psi+v, \theta, t)
$$

and following "reflection" map

$$
R^{\prime}((\psi, \theta, t))=(-\psi, R(\theta, t))=(-\psi,-\theta,-t) .
$$

is an isometric involution.

We denote the universal cover of $\Omega$ by $\widehat{\Omega}$. That is,

$$
\widehat{\Omega}=\mathbb{R} \times \mathbb{R} \times \mathbb{R}
$$

with coordinates $(\widehat{\psi}, \widehat{\theta}, \widehat{t})$. Let $\widehat{\Pi}: \widehat{\Omega} \rightarrow \Omega$ be the natural covering map. For subsets $S \subset \Omega$ we will denote lifts of these sets to $\widehat{\Omega}$ by $\widehat{S}$. In particular, the tori $\mathbb{T}^{2}[ \pm 1]$ lift to stable minimal disks $\widehat{\mathbb{T}}^{2}[ \pm 1]$ and the cylinders $\Gamma_{ \pm}$lift to minimal disks $\widehat{\Gamma}_{ \pm}$ which together bound a region $\widehat{V}:=\widehat{V}^{\prime}$ which contains $(0,0,0)$. Likewise, we let $\widehat{A}_{i}=\mathbb{R} \times\{\pi+2 \pi i\} \times(-1,1)$ for $i \in \mathbb{Z}$ be lifts of $A$. We denote by $\widehat{T}_{v}$ the isometry of $\widehat{\Omega}$ given by $\widehat{T}_{v}^{1}(\widehat{\psi}, \widehat{\theta}, \widehat{t})=(\widehat{\psi}+v, \widehat{\theta}, \widehat{t})$ for $v \in \mathbb{R}$ and let $\widehat{R}$ be the reflection $\widehat{R}(\widehat{\psi}, \widehat{\theta}, \widehat{t})=(-\widehat{\psi},-\widehat{\theta},-\widehat{t})$. Note that $\widehat{T}_{2 \pi i}$ for $i \in \mathbb{Z}$ are deck transforms of the cover. Furthermore, $\widehat{R}\left(\widehat{\Gamma}^{+}\right)=\widehat{\Gamma}^{-}$and $\widehat{R}\left(\widehat{A}_{i}\right)=\widehat{A}_{-i-1}$. Finally, let us denote by $\widehat{G}_{i}, i \in \mathbb{Z}$, the deck transforms

$$
\widehat{G}_{i}((\psi, \theta, t))=(\psi, \theta+2 \pi i, t)
$$

and note that $\widehat{G}_{i}(\widehat{V}) \cap \widehat{V}=\emptyset$ for $i \in \mathbb{Z} \backslash\{0\}$.

We now construct an embedded minimal disk $\widehat{\Delta}$ in $\widehat{\Omega}$ so that $\Delta=\widehat{\Pi}(\widehat{\Delta})$. To that end, let $\widehat{\sigma}_{j}^{+}$be the curves $\widehat{\Gamma}_{+} \cap\{\widehat{\psi}=j\}$ and $\widehat{\sigma}_{j}^{-}$the curves $\widehat{\Gamma}_{-} \cap\{\widehat{\psi}=-j\}$. We denote by $\widehat{\sigma}_{j, i}^{+}$the segment of $\widehat{\sigma}_{j}$ between $\widehat{A}_{i}$ and $\widehat{A}_{-i-1}$ and likewise for $\widehat{\sigma}_{j, i}^{-}$. Let $\widehat{\tau}_{j, i}^{-}$be a real-analytic curve connecting the endpoint of $\widehat{\sigma}_{j, i}^{+}$in $\widehat{A}_{-i-1}$ to the endpoint of $\widehat{\sigma}_{j, i}^{-}$which is chosen to be contained in $\widehat{A}_{-i-1}$ and to have the property 
that both coordinates $\widehat{t}$ and $\widehat{\psi}$ are strictly monotonic. Set $\widehat{\tau}_{j, i}^{+}=\widehat{R}\left(\widehat{\tau}_{j, i}^{-}\right)$. One verifies that $\widehat{\tau}_{j, i}^{+}$connects the other endpoints of $\widehat{\sigma}_{j, i}^{+}$and $\widehat{\sigma}_{j, i}^{-}$. Hence,

$$
\widehat{\delta}_{j, i}:=\widehat{\sigma}_{j, i}^{+} \cup \widehat{\sigma}_{j, i}^{-} \cup \tau_{j, i}^{+} \cup \tau_{j, i}^{-}
$$

is a closed curve and $\widehat{\delta}_{j, i}=\widehat{R}\left(\widehat{\delta}_{j, i}\right)$. We note that our choice of curves implies further that $\widehat{T}_{v}\left(\widehat{\delta}_{j, i}\right) \cap \widehat{\delta}_{j, i}=\emptyset$ for $v \neq 0$. Now let $\widehat{\Delta}_{j, i}$ be minimal disks which solve the Plateau problem with boundary $\widehat{\delta}_{j, i}$. By the strong maximum principle $\widehat{T}_{v}^{1}\left(\widehat{\Delta}_{j, i}\right) \cap \widehat{\Delta}_{j, i}=\emptyset$ for $v \neq 0$. In particular, $\left\{\widehat{T}_{v}^{1}\left(\widehat{\Delta}_{j, i}\right)\right\}_{v \in \mathbb{R}}$ is a minimal foliation $\mathcal{D}_{j, i}$ of an open subset, $\widehat{V}_{j, i}$, of $\widehat{V}$ and $\widehat{T}_{v}$ leaves $\mathcal{D}_{j, i}$ invariant. This together with the strong maximum principle applied to the Jacobi function generated by $\widehat{T}_{v}$ implies that the leaves of $\mathcal{D}_{j, i}$ are graphs over

$$
V_{i}:=\{(\widehat{\psi}, \widehat{\theta}, \widehat{t}) \in \widehat{V}: \widehat{\psi}=0, \theta \in(-\pi-2 \pi i, \pi+2 \pi i)\} .
$$

As $\widehat{R}$, leaves both $\widehat{\delta}_{j, i}$ and $\widehat{V}_{j, i}$ unchanged, it follows from the strong maximum principle that $\widehat{\Delta}_{j, i}=\widehat{R}\left(\widehat{\Delta}_{j, i}\right)$. In particular $(0,0,0) \in \widehat{\Delta}_{j, i}$. By Theorem 3.5. up to passing to a subsequence, the minimal foliations $\mathcal{D}_{j, i}$ converge smoothly on compact subsets of $\widehat{V}$ to a minimal foliation of $\widehat{V}$. This foliation is also invariant under $\widehat{T}_{v}$. A consequence of this is that if $L$ is a leaf of $\mathcal{D}$, then either $L$ splits as the product $\mathbb{R} \times \eta$ where $\eta$ is a geodesic in $V_{\infty}$ or $L$ is graph over some open subset of $V_{\infty}$. If the former occurs, then the stability of $L$ implies that $\eta$ is also a stable geodesic.

Let $\widehat{\Delta}$ be the leaf of $\mathcal{D}$ which contains $(0,0,0)$. As $\widehat{\Delta}$ is the limit of $\widehat{\Delta}_{j, i}$, $\widehat{\Delta}$ is complete. By Lemma A.1, the geodesic in $V_{\infty}$ through $(0,0,0)$ is unstable and hence $\widehat{\Delta}$ cannot split. In particular, $\widehat{\Delta}$ is a graph over some open subset of $V_{\infty}$ and $\widehat{T}_{v}(\widehat{\Delta}) \cap \widehat{\Delta}=\emptyset$ Set $\Delta=\widehat{\Pi}(\widehat{\Delta})$ and note that, as $\widehat{\Delta} \subset \widehat{V}, \widehat{G}_{i}(\widehat{\Delta}) \cap$ $\widehat{\Delta}=\emptyset$ for $i \in \mathbb{Z} \backslash\{0\}$. Hence, $\Delta$ is a complete embedded minimal disk in $V \subset$ $\Omega$. Clearly $\Delta$ cannot be properly embedded in $\Omega$. Nevertheless, the curvature estimates for stable minimal surfaces imply that $\bar{\Delta}$ is a smooth minimal lamination in $\Omega$. To determine the other leaves we note first that $\lim _{v \rightarrow \infty} \widehat{T}_{v}(\Delta)$ converges in $\widehat{\Omega}$ to some $\widehat{T}_{v}$ invariant minimal surface $\widehat{L}_{+}-$possibly, but not necessarily, $\widehat{\Gamma}_{+}$. Similarly, $\lim _{v \rightarrow-\infty} \widehat{T}_{v}(\widehat{\Delta})$ converges to a $\widehat{T}_{v}$ invariant surface $\widehat{L}_{-}=\widehat{R}\left(\widehat{L}_{+}\right)$. As a consequence, $L_{ \pm}=\widehat{\Pi}\left(\widehat{L}_{ \pm}\right)$are non-proper embedded minimal annuli that are leaves of $\bar{\Delta}$. Finally, by construction $L_{ \pm}$are contained in $\bar{V}^{\prime}$, that is are trapped between $\Gamma_{+}$and $\Gamma_{-}$. Since the ends of $\Gamma_{+}$and $\Gamma_{-}$converge to the same side of $\mathbb{T}_{1}^{2}$ and $\mathbb{T}_{-1}^{2}$, one verifies that $\bar{L}_{ \pm}=\mathbb{T}^{2}[ \pm 1]$ and these are the remaining leaves of $\bar{\Delta}$. That is, $\Delta=\widehat{\Pi}(\widehat{\Delta})$ is the desired minimal disk.

\section{Appendix B. Curvature Estimate of Schoen And Simon}

For the convenience of the reader we state the curvature estimate for embedded minimal disks with a uniform area bound proved by Schoen and Simon in [19]. 
Theorem B.1. Fix $(\Omega, g)$ a Riemannian three-manifold and let $\mathcal{B}_{2 r}(p) \subset N$ satisfy:

- $\exp _{p}^{\Omega}: B_{2 r}(0) \rightarrow \mathcal{B}_{2 r}(p)$ is a smooth diffeomorphism;

- With $g_{i j} d x^{i} d x^{j}=\left(\exp _{p}^{\Omega}\right)^{*}$ g, there is an $0<\alpha \leq 1$ so

$$
\frac{1}{2} \delta_{i j}<g_{i j}<2 \delta_{i j}, \sup _{B_{2 r}}\left(r\left|\frac{\partial g_{j k}}{\partial x^{i}}\right|+r^{2}\left|\frac{\partial^{2} g_{k l}}{\partial x^{i} \partial x^{j}}\right|\right)<1,
$$

and

$$
\sup _{(x, y) \in B_{2 r} \times B_{2 r}} r^{2+\alpha}|x-y|^{-\alpha}\left|\frac{\partial^{2} g_{k l}}{\partial x^{i} \partial x^{j}}(x)-\frac{\partial^{2} g_{k l}}{\partial x^{i} \partial x^{j}}(y)\right|<1 .
$$

Given $\mu>0$, there is a $C=C(\mu)>0$ so that if $\Sigma \subset \mathcal{B}_{2 r}(p)$ is a properly embedded minimal disk in $\mathcal{B}_{2 r}(p)$ and $|\Sigma| \leq \mu r^{2}$, then

$$
\sup _{\mathcal{B}_{r}(p) \cap \Sigma}|A|^{2}<C r^{-2} \text {. }
$$

\section{REFERENCES}

[1] M. Anderson, Curvature estimates for minimal surfaces in 3-manifolds, Ann. Scient. Éc. Norm. Sup. 18 (1985), 89-105, MR0803196, Zbl 0578.49027.

[2] J. Bernstein and C. Breiner, Helicoid-like minimal disks and uniqueness, J. Reine Angew. Math. 655 (2011), 129-146, MR2806108, Zbl 1225.53008.

[3] M. Calle and D. Lee, Non-proper helicoid-like limits of closed minimal surfaces in 3-manifolds, Math. Z. (2009), 1-12, MR2480755, Zbl 1161.53043.

[4] T. H. Colding and W. P. Minicozzi II, Embedded minimal disks: proper versus nonproper global versus local, Transactions of A.M.S. 356 (2003), no. 1, 283-289, MR2020033, Zbl 1046.53001.

[5] _ The space of embedded minimal surfaces of fixed genus in a 3-manifold I; Estimates off the axis for disks, Annals of Math. 160 (2004), 27-68, MR2119717, Zbl 1070.53031.

[6] _ The space of embedded minimal surfaces of fixed genus in a 3-manifold II; Multivalued graphs in disks, Annals of Math. 160 (2004), 69-92, MR2119718, Zbl 1070.53032.

[7] _ The space of embedded minimal surfaces of fixed genus in a 3-manifold III; Planar domains, Annals of Math. 160 (2004), 523-572, MR2123932, Zbl 1076.53068.

[8] _ The space of embedded minimal surfaces of fixed genus in a 3-manifold IV; Locally simply-connected, Annals of Math. 160 (2004), 573-615, MR2123933, Zbl 1076.53069.

[9] B. Dean, Embedded minimal disks with prescribed curvature blowup, Proc. Amer. Math. Soc. 134 (2006), no. 4, 1197-1204, MR2196057, Zbl 1100.53009.

[10] D. Hoffman and B. White, Limiting behavior of sequences of properly embedded minimal disks, In preparation.

[11] _ Sequences of embedded minimal disks whose curvatures blow up on a prescribed subset of a line, Comm. Anal. Geom. 19 (2011), no. 3, 487-502, MR2843239, Zbl 1244.53010.

[12] S. Khan, A minimal lamination of the unit ball with singularities along a line segment, Illinois J. Math. 53 (2009), no. 3, 833-855 (2010), MR2727357, Zbl 1225.53009.

[13] S. J. Kleene, A minimal lamination with Cantor set-like singularities, Proc. Amer. Math. Soc. 140 (2012), no. 4, 1423-1436, MR2869127, Zbl 1239.53006.

[14] W. H. Meeks III, Regularity of the singular set in the Colding and Minicozzi lamination theorem, Duke Math. J. 123 (2004), no. 2, 329-334, MR2066941, Zbl 1086.53005.

[15] _ The limit lamination metric for a Colding-Minicozzi minimal lamination, Illinois J. of Math. 49 (2005), no. 2, 645-658, MR2164355, Zbl 1087.53058.

[16] W. H. Meeks III and H. Rosenberg, The uniqueness of the helicoid, Annals of Math. 161 (2005), 723-754, MR2153399, Zbl 1102.53005. 
[17] W. H. Meeks III and M. Weber, Bending the helicoid, Math. Ann. 339 (2007), no. 4, 783-798, MR2341900, Zbl 1156.53011.

[18] W. H. Meeks III and S. T. Yau, The existence of embedded minimal surfaces and the problem of uniqueness, Math. Z. 179 (1982), 151-168, MR0645492, Zbl 0479.49026 Math. Z. 179, 151-168 (1982)..

[19] R. M. Schoen and L. Simon, Regularity of simply connected surfaces with quasiconformal Gauss map, in Seminar on Minimal Submanifolds, Ann. of Math. Stud., Princeton University Press, 1983, 127-145, MR0795232, Zbl 0544.53001.

[20] L. Simon, Lectures on geometric measure theory, Proceedings of the Center for Mathematical Analysis (Canberra, Australia), vol. 3, Australian National University, 1983, MR0756417, Zbl 0546.49019.

[21] B. Solomon, On foliations of $\mathbb{R}^{n+1}$ by minimal hypersurfaces, Comm. Math. Helv. 61 (1986), 67-83, MR0847521, Zbl 0601.53025.

[22] B. White, Curvatures of embedded minimal disks blow up on subsets of $C^{1}$ curves, preprint, arXiv:1103.5551

[23] _ Curvature estimates and compactness theorems in 3-manifolds for surfaces that are stationary for parametric elliptic functionals, Invent. Math. 88 (1987), no. 2, 243-256, MR0880951, Zbl 0615.53044.

[24] Which ambient spaces admit isoperimetric inequalities for submanifolds?, J. Differential Geometry 83 (2009), no. 1, 213-228, MR2545035, Zbl 1179.53061. 